\title{
A Novel Distributed Self-Protection Scheme for WDM-PON Using a Circulator-AWG Based Wavelength Router
}

\author{
Xiaofei Cheng, Yong-kee Yeo, Xu Shao, Yixin Wang \\ RF and Optical Department \\ Institute for Infocomm Research $\left(\mathrm{I}^{2} \mathrm{R}\right), \mathrm{A}^{*} \mathrm{STAR}$, \\ 1 FusionoPolis Way, \#21-01, Connexis, \\ Singapore 138632 \\ chengxf@i2r.a-star.edu.sg
}

\author{
Bai Yaohui \\ Jiangxi University of Finance and Economics \\ byhnu@163.com
}

\begin{abstract}
We propose a novel distributed self-protection scheme for WDM-PONs using circulator-AWG based wavelength router. The novel wavelength router enables proper routing of working and protection lightpaths of the uplink and downlink signals. Automatic protection switching is operated in a distributed control manner and thus ultra-short $0.7-\mu$ s protection switching time is achieved. Redundant fibers required for protection are minimized.
\end{abstract}

Keywords- WDM-PON; protection; Survivability

\section{INTRODUCTION}

Wavelength division multiplexed passive optical network (WDM-PON) has been considered as promising solution for future broadband access networks. With the rapid increase of transmission capacity of WDM-PONs, survivability becomes significant since any fiber failure may disrupt a large amount of transmission data. Recently, protection schemes for WDMPONs have been extensively studied [1-5]. In [1-3], feeder fibers are duplicated for protection, while ONUs are either adjacently connected $[1,2]$ or fully connected to form a ring [3] to provide redundant network resources against distribution fiber failures. In [4], both feeder fiber and distribution fibers are duplicated for protection. In [5], we proposed a combined dual-PON protection scheme whereby two PONs provide mutual protection for each other.

In this paper, we propose a novel distributed selfprotection scheme for WDM-PONs using circulator-arrayed waveguide grating (circulator-AWG) based wavelength router at remote node $(\mathrm{RN})$. The novel wavelength router enables proper routing of working and protection lightpaths of the uplink and downlink signals. Automatic protection switching is operated in a distributed control manner and thus ultra-short $0.7-\mu$ s protection switching time is achieved. Compared with previous schemes [2-5], redundant fibers required for protection are minimized to reduce fiber installation or rental cost. The power penalty due to performance degradation from the protection path is negligible.

\section{NOVEL RELIABLE WDM-PON ARCHITECTURE}

Fig.1 shows our proposed network architecture. In the central office (CO), downlink and uplink channels are multiplexed/demultiplexed by an $N \times 1$ multiplexer (MUX) and a $1 \times N$ cyclic AWG, respectively. A 3-port circulator is used to separate the uplink and downlink signals. The multiplexed downlink signals are duplicated via a 3-dB fiber coupler and sent over both a working and a protection feeder fiber to the $\mathrm{RN}$. The RN consists of two 3-port full circulators and an $N \times N$ cyclic (AWG). At ONU side, all ONUs are numbered in sequence. We group two ONUs of $\mathrm{ONU}_{k}$ and $\mathrm{ONU}_{N / 2+k}$ as an ONU pair where $1 \leq k \leq N / 2$ and connect each ONU pair with a connection fiber (CF). Note that the two ONUs in a pair are physically located adjacent, so that the CF could be relatively short. Two blue/red (B/R) filters are used to separate/combine the uplink and downlink channels of the local ONU and the counterpart $\mathrm{ONU}$ in an $\mathrm{ONU}$ pair. At $\mathrm{ONU}_{k}$, a small portion of the downlink signal power from port B (for $1 \leq k \leq N / 2$ ) or R (for $N / 2+1 \leq k \leq N$ ) of the left B/R filter (See Fig.1(a)) is tapped to a monitoring and control (MC) circuit, which is responsible for monitoring failure and controlling the local optical switch (OS) state. The OS in each ONU is initially set for port 1-2 connection under normal situation.

In order to eliminate fiber Rayleigh backscattering, we propose a novel wavelength assignment scheme which the downlink and uplink signals occupy different wave bands with a wavelength shift of FSR/2, where FSR is the free spectral range of the $N \times N$ cyclic AWG. Under this scheme, only one $\mathrm{CF}$ fiber is required to connect each ONU pair replace of the two CF fibers required in previous schemes. The number of rredundant fibers required for protection is thus minimized. 


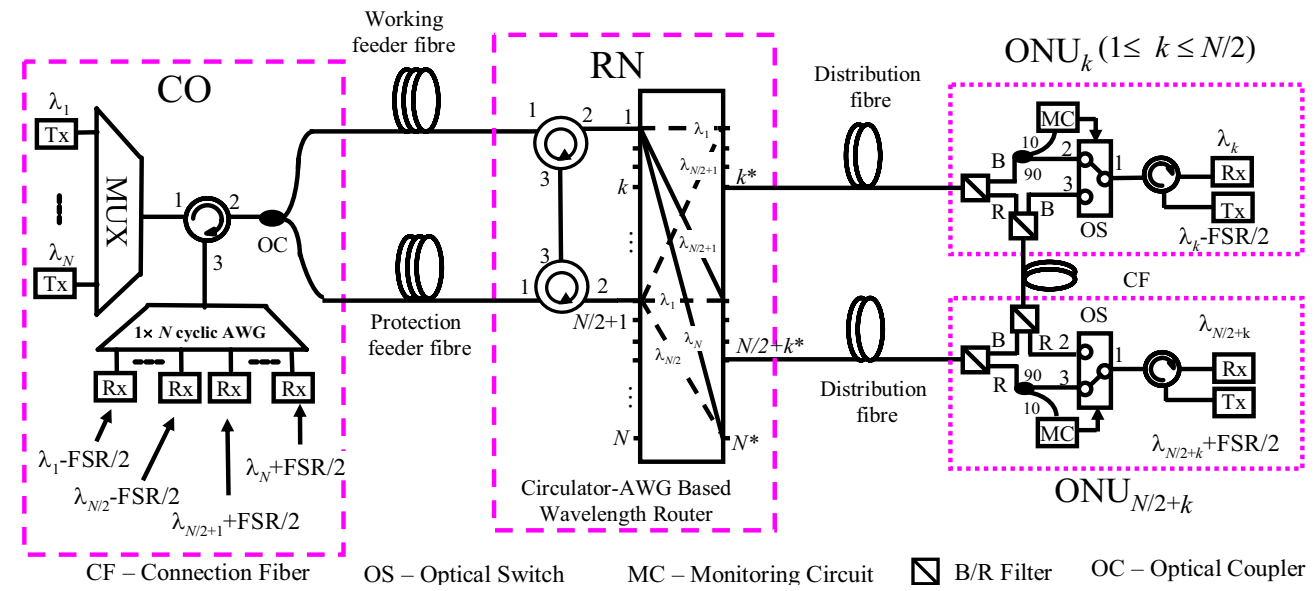

Fig. 1 Proposed reliable WDM-PON architecture using (a) two 3-port full circulators

The wavelength assignment scheme is shown in Fig. 2. Downlink wavelengths of $\mathrm{ONU}_{1}$ to $\mathrm{ONU}_{N}$ are assigned with wavelengths $\lambda_{1}$ to $\lambda_{N}$. Note that $\lambda_{1}$ will appear at port $k^{*}$ $(1 \leq k \leq N)$ when it is fed into port $k$ of the $N \times N$ cyclic AWG. The uplink wavelength of $\mathrm{ONU}_{k}(1 \leq k \leq N / 2)$ is $\lambda_{k}$-FSR/2. While for $N / 2+1 \leq k \leq N$, the corresponding uplink wavelength is $\lambda_{k}+\mathrm{FSR} / 2$.

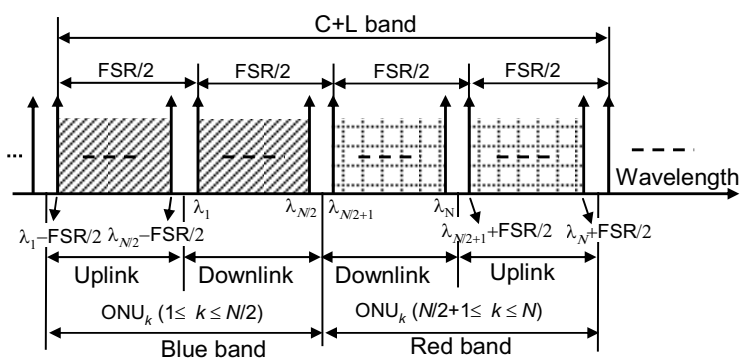

Fig. 2 Proposed wavelength assignment scheme

Fig. 3 shows routing scheme of working and protection wavelength channels for traffics of $\mathrm{ONU}_{k}$. In normal state, downlink signal of $\mathrm{ONU}_{k}$ is routed to the output port $k^{*}$ of the AWG at the RN via the working feeder fiber and the upper 3port circulator at the $\mathrm{RN}$, and is detected by the receiver $(\mathrm{Rx})$ at $\mathrm{ONU}_{k}$ after passing through the distribution fiber and the $\mathrm{B}$ (for $1 \leq k \leq N / 2$ ) or $\mathrm{R}$ (for $N / 2+1 \leq k \leq N$ ) port of the $\mathrm{B} / \mathrm{R}$ filter at $\mathrm{ONU}_{k}$. While uplink signal of $\mathrm{ONU}_{k}$ is routed to input port $N / 2+1$ of the AWG at the RN via the same filter and the distribution fiber and is detected at the $\mathrm{CO}$ after passing through all two 3-port circulators at the $\mathrm{RN}$ and the working feeder fiber. When fiber failure occurs, which results in low or no downlink signal power in $\mathrm{ONU}_{k}$, the local OS is triggered to 1-3 connection by the corresponding $\mathrm{MC}$ circuit. In this case, the downlink signal of $\mathrm{ONU}_{k}$ is routed to output port $N / 2+k^{*}$ of the AWG at the RN via the protection feeder fiber and the lower circulator at the $\mathrm{RN}$, and is detected in $\mathrm{ONU}_{k}$ via the distribution fiber of $\mathrm{ONU}_{N / 2+k}$ and $\mathrm{B}$ (for $1 \leq k \leq N / 2$ ) or R (for $N / 2+1 \leq k \leq N$ ) port of the $\mathrm{B} / \mathrm{R}$ filters in the counterpart $\mathrm{ONU}_{N / 2+k}$ and $\mathrm{ONU}_{k}$, as well the connection fiber. While the uplink signal is routed to input port 1 of the AWG at the RN via the same filters and the distribution fiber, and is detected in the $\mathrm{CO}$ after passing through the two 3-port full circulator at the $\mathrm{RN}$ and the protection feeder fiber.

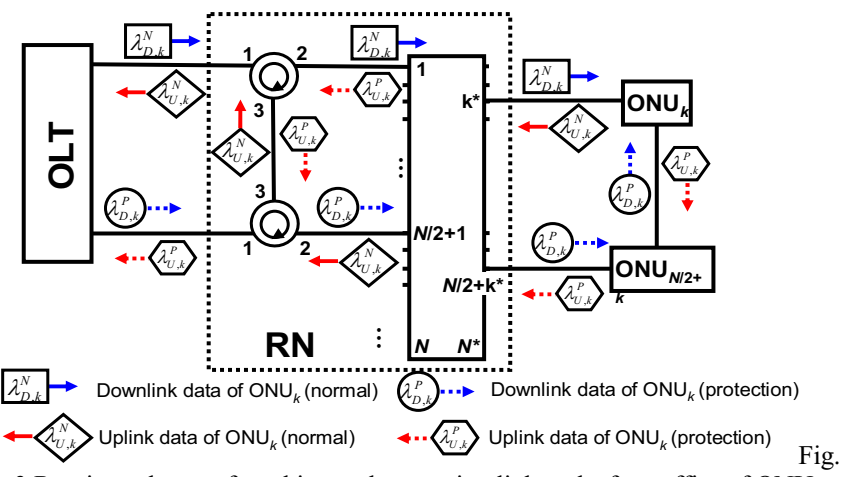

3 Routing scheme of working and protection lightpaths for traffics of $\mathrm{ONU}_{\mathrm{k}}$

Table 1 shows the detailed wavelength routing scheme of the $N \times N$ cyclic AWG at the RN. In the normal state, downlink signal of $\mathrm{ONU}_{k}$ is routed to the output port $k^{*}$ of the AWG at the RN via the working feeder fiber and is detected by the receiver ( $\mathrm{Rx})$ at $\mathrm{ONU}_{k}$ after passing through the distribution fiber and the B (for $1 \leq k \leq N / 2$ ) or R (for $N / 2+1 \leq k \leq N$ ) port of the $\mathrm{B} / \mathrm{R}$ WDM coupler at $\mathrm{ONU}_{k}$. While uplink signal of $\mathrm{ONU}_{k}$ is routed to output port $N / 2+1$ of the AWG via the same B or R port of the coupler and the distribution fiber and is detected in the $\mathrm{CO}$ after passing through the two full circulators at the $\mathrm{RN}$ and the working feeder fiber. When fiber failure occurs, resulting in low or no downlink signal power in $\mathrm{ONU}_{k}$, the local OS is triggered to 1-3 connection by the corresponding $\mathrm{MC}$ circuit. Both downlink and uplink signals of $\mathrm{ONU}_{k}$ are transmitted through their protection path. In this case, the downlink signal of $\mathrm{ONU}_{k}$ is routed to output port $N / 2+k^{*}$ of the $\mathrm{AWG}$ at the RN through the protection feeder fiber and is detected in $\mathrm{ONU}_{k}$ after passing through the distribution fiber of $\mathrm{ONU}_{N / 2+k}$ and the B (for $1 \leq k \leq N / 2$ ) or R (for $N / 2+1 \leq k \leq N$ ) port of the $\mathrm{B} / \mathrm{R}$ WDM couplers in $\mathrm{ONU}_{k}$ and the counterpart $\mathrm{ONU}_{N / 2+k}$. While the uplink signal is routed to output port 1 of the AWG via the same $B$ or $R$ port of the couplers and the same distribution fiber and is then detected in the $\mathrm{CO}$ after passing through the two full circulators at the $\mathrm{RN}$ and the protection feeder fiber. 


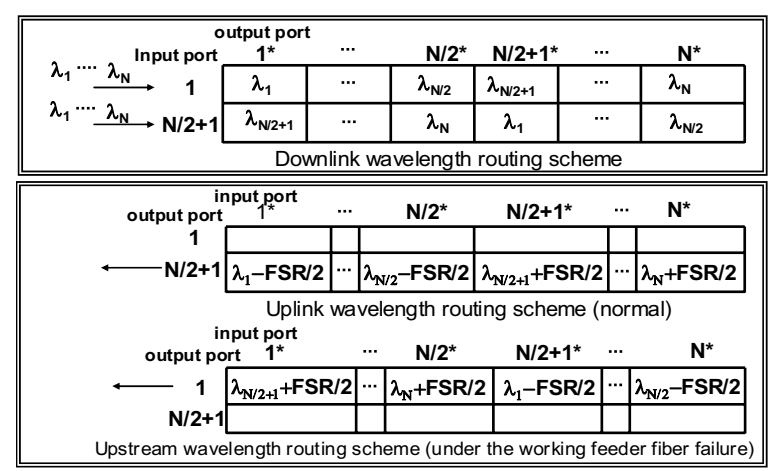

Table 1 Wavelength routing scheme of the AWG at RN

\section{EXPERIMENT SETUP}

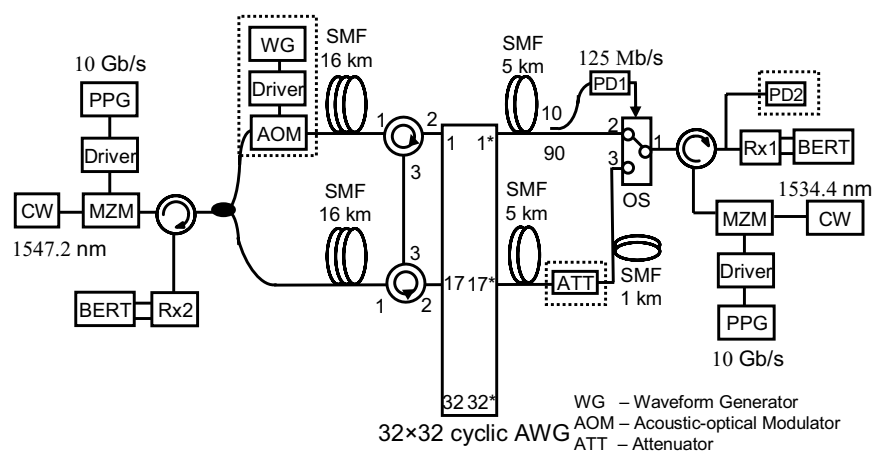

Fig. 3 Experiment setup

Fig. 4 shows the experimental setup. The $10-\mathrm{Gb} / \mathrm{s}$ signals for both downlink and uplink were generated by externally modulating a continuous-wave (CW) light using a MachZehnder modulator (MZM). The wavelengths of downlink and uplink signals were $1547.2 \mathrm{~nm}$ and $1534.4 \mathrm{~nm}$, respectively. The pseudo-random bit sequence with a word length of $2^{23}-1$ used to drive the MZM was provided by a pulse pattern generator (PPG). A commercial $32 \times 32$ cyclic AWG with $100 \mathrm{GHz}$ channel spacing and an FSR of $25.6 \mathrm{~nm}$ was used at the RN. Both the feeder fiber and the distribution fiber were single mode fiber (SMF) with lengths of $16 \mathrm{~km}$ and $5 \mathrm{~km}$, respectively. The CF was a $1-\mathrm{km}$ SMF. The downlink signal was detected by a $10-\mathrm{GHz}$ receiver $(\mathrm{Rx} 1)$ at the ONU side. The $10-\mathrm{Gb} / \mathrm{s}$ uplink signal was detected at the CO by another $10-\mathrm{GHz}$ receiver $(\mathrm{Rx} 2)$. The optical switch in our experiment was a commercial AGILTRON Nano Second $2 \times 2$ optical switch with $1.2-\mathrm{dB}$ insertion loss and $23-\mathrm{dB}$ crosstalk. In order to emulate the fiber failure scenario, an acoustic-optic modulator (AOM) was inserted just before the working feeder fiber. The AOM was driven by a square waveform from a waveform generator (WG) with a repetition rate of $1 \mathrm{kHz}$. A NEW FOCUS 125-MHz photo detector (PD1) was used to detect the on-off state of the working link. The resulting PD1 output was used to control the optical switch. Another NEW

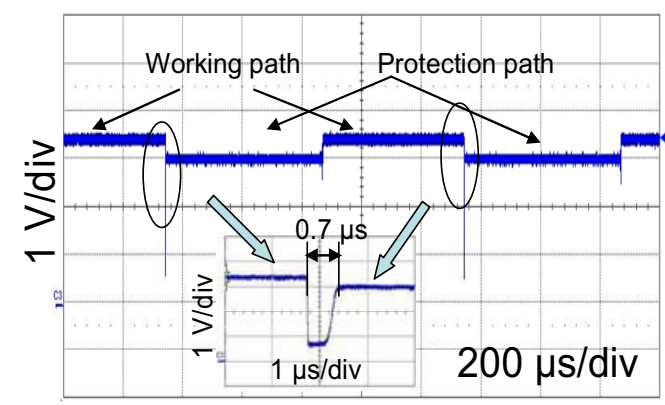

Fig. 5 Measured protection switching time

FOCUS 125-MHz photo detector (PD2) was used after the circulator in the $\mathrm{ONU}$ to monitor the protection switching time.

Fig. 5 shows the measured protection switching time for the downlink signal. The upper and lower traces represent the signals received from the working and protection paths, respectively. As shown in Fig. 5, the protection build-up time is $0.7 \mu \mathrm{s}$. Local rather than remote signalling delivery contributed to the fast protection time due to the distributed protection scheme. The ultra-short switching time of the OS used in the experiment was also contributed to the short buildup time.

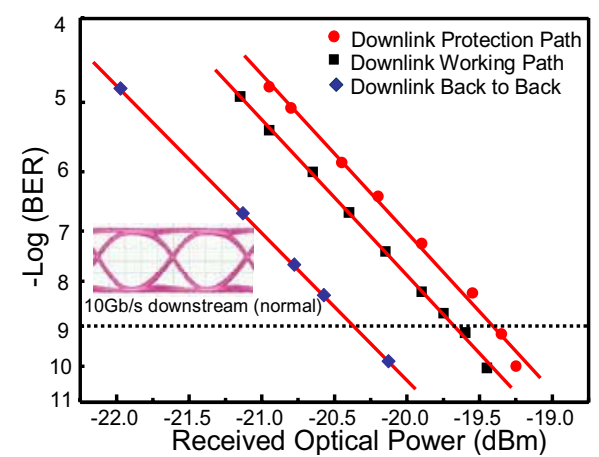

(a)

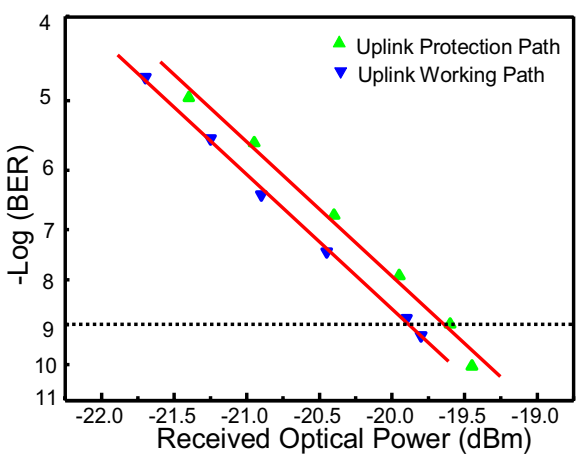

(b)

Fig. 6 Measured BER curves of (a) 10-Gb/s downlink signal and (b) 10-Gb/s uplink signal on both working and protection paths

Fig. 6 shows the measured BER curves for the $10-\mathrm{Gb} / \mathrm{s}$ uplink and downlink signals. The receiver sensitivity for the working path is slightly better than that of the protection path. The power penalty due to the performance degradation of the 
protection path is $0.3 \mathrm{~dB}$ and is negligible.

\section{CONCLUSIONS}

We have proposed a novel distributed self-protection scheme for WDM-PON using novel circulator-AWG based wavelength router. The number of extra fibers required for protection is minimized to reduce the fiber installation or rental cost. The power penalty due to the performance degradation of the protection path is $0.3 \mathrm{~dB}$. A short protection build-up time of $0.7 \mu$ s was observed using this distributed protection manner. Both downlink and uplink transmissions at $10-\mathrm{Gb} / \mathrm{s}$ were evaluated to verify the proposed scheme.

\section{REFERENCES}

[1] T. J. Chan et al, "A novel bidirectional wavelength division multiplexed passive optical network with 1:1 protection", OFC, FQ6, 779-781(2003).

[2] Z. Wang et al "A broadcast and select WDM-PON and its protection," ECOC, We4.P.024, 549-550 (2005).

[3] X. Sun et al,"A novel star-ring protection architecture scheme for WDM passive optical access networks," OFC, JWA53 (2004).

[4] K. Lee, et al, "Reliable Wavelength-Division-Multiplexed Passive Optical Network Using Novel Protection Scheme," PTL 20, 679-781 (2008).

[5] X. F. Cheng et al, "A Novel Protection Scheme for WDM Passive Optical network," in Proc. ECOC, WP3.P.151 (2006). 